\title{
Motivational Game Design Patterns of 'Ville Games
}

\author{
Chris Lewis, Noah Wardrip-Fruin, Jim Whitehead \\ University of California, Santa Cruz \\ 1156 High St, Santa Cruz, California, USA \\ \{cflewis,nwf,ejw\}@soe.ucsc.edu
}

\begin{abstract}
The phenomenal growth of social network games in the last five years has left many game designers, game scholars, and long-time game players wondering how these games so effectively engage their audiences. Without a strong understanding of the sources of appeal of social network games, and how they relate to the appeal of past games and other human activities, it has proven difficult to interpret the phenomenon accurately or build upon its successes. In this paper we propose and employ a particular approach to this challenge, analyzing the motivational game design patterns in the popular 'Ville style of game using the lenses of behavioral economics and behavioral psychology, explaining ways these games engage and retain players. We show how such games employ strategies in central, visible ways that are also present (if perhaps harder to perceive) in games with very different mechanics and audiences. Our conclusions point to lessons for game design, game interpretation, and the design of engaging software of any type.
\end{abstract}

\section{Categories and Subject Descriptors}

D.m [Software]: Miscellaneous-Software psychology; K.8.0 [Personal Computing]: General_Games; H.5 [Information Interfaces and Presentation]: User Interfaces

\section{Keywords}

social network games, behavioral economics, behavioral psychology, design patterns

\section{INTRODUCTION}

In July 2007, Mark Pincus set up Zynga, a social network game (SNG) company. The company now serves 246 million players, primarily through their flagship 'Ville series. The success of SNGs has surprised the game design community, prompting a widely discussed talk by Jesse Schell, pronouncing, "Everyone's been talking Facebook, Facebook,

Permission to make digital or hard copies of all or part of this work for personal or classroom use is granted without fee provided that copies are not made or distributed for profit or commercial advantage and that copies bear this notice and the full citation on the first page. To copy otherwise, to republish, to post on servers or to redistribute to lists, requires prior specific permission and/or a fee.

FDG '12 May 29 - June 1, 2012 Raleigh, NC, USA.

Copyright 2012 ACM 978-1-4503-1333-9/12/05 ...\$10.00.
Facebook. . Facebook knocked us on our collective ass this year. I don't think a lot of us saw it coming" [29].

One peculiar aspect of many SNGs is that they appear to defy the current understanding of what a good game actually is. Sid Meier theorizes that a good game "is a series of interesting choices" [28], Raph Koster believes that "with games, learning is the drug" [19], while Jane McGonigal posits that engaging games stem from positive stress (otherwise known as hard fun) [23]. Do SNGs really do any of these things?

Players play SNGs in a casual, relaxed manner, tending to and decorating a virtual space, and performing quests that unlock new items. Usually these games do not contain any immediate threats or challenges that cannot wait. Many available game actions do not provide much consequence, which is likely a requirement for a choice to be interesting. Often, the gameplay systems of SNGs can be understood quickly, allowing all players to adapt within one or two play sessions. The casual pace of game play in most SNGs does not often engender a feeling of stress.

These games appear to be outside the boundaries of current models of game understanding, yet they have led to an industry which engages a large and varied audience [1]. Perhaps most telling of how little we understand SNGs is the success of Cow Clicker, a parody of FarmVille by Ian Bogost, which itself gained 50000 players. The goal of the game was to click your cow more than anyone else. When Bogost's malevolent attempts to ruin the game, such as switching the default cow to another direction and charging $\$ 20$ didn't work (people bought it), he removed all the cows, leading to complaints that the game without cows was "not a very fun game" [36].

In this paper, we analyze the "Ville style of games for specific game design patterns that motivate player behavior, which we term "motivational game design patterns." We connect these patterns to behavioral economics, a field dedicated to the study of decision-making, and behavioral psychology, a field dedicated to discovering our innate reactions to given situations. Behavioral economics has provided many theories as to why broad populations of people repeatedly make certain decisions. We link the theories from behavioral economics to the patterns we present, providing possible explanations as to why players are motivated to return to SNGs.

The patterns we present are relatively well-known with the social game design community and occur often, and some developers may well have made linkages between these patterns and behavioral economics and behavioral psychology, but, to our knowledge, neither the patterns nor the science 
behind them have been throughly documented.

We limit our analysis to 'Ville games because, as Wohn et al. note, "what constitutes a SNG is determined more by the technical aspects of how it is accessed and distributed, not on the genre of game" [37]. We chose to study "Ville games based on their continued popularity, with Zynga easily commanding the most players at the time of writing ${ }^{1}$.

To evaluate our approach, we attempt to use our framework to provide an explanation of Cow Clicker's popularity. The bare-bones design, removing any other game design elements that may have accidentally led to the game being enjoyable, provides a strong test subject for our framework.

\section{BACKGROUND THEORY}

\subsection{Overview}

Throughout this paper, we will use a number of different theories from behavioral economics and behavioral psychology, some of which appear in multiple design patterns. In this section, we will define these theories, so that readers can easily refer back to them.

\subsection{Behavioral Economics}

Behavioral economics is a field which utilizes cognitive psychology to analyze peoples' decision-making, and takes particular interest in anomalies from the standard economic model, where people do not act "rationally" (i.e. a person makes some decision which does not lead to a maximum gain for herself) [22].

\subsubsection{Anchoring}

In the absence of knowing what something is worth, we will be accept the first price we see as being the baseline. This is known as anchoring: we become anchored to that price [4]. All our judgments of value are then based on whether something appears relatively cheaper or more expensive.

\subsubsection{Contrast effect}

The contrast effect describes how people have a tendency to perceive things relativistically [35]. We can see this in games often: a starting car feels moderately powerful in a driving game, but once you're used to the supercars, the starting feels much slower than it did before.

\subsubsection{Endowment progress effect}

The endowment progress effect builds upon the findings of goal-gradient hypothesis (described later in 2.3.2), showing that simply the illusion of progress is enough to induce accelerated behavior; so if a 12-stamp coffee card is given with the first two marked off for free, customers purchase the final 10 coffees quicker than if a 10-stamp card is used [18, 24]. Hamari theorizes World of Warcraft's 'rested' bonus creates this effect [12].

\subsubsection{Hedonic treadmill}

The hedonic treadmill reflects the process of continually striving towards a goal that a person anticipates will make them happy, but then their happiness returns to a baseline,

\footnotetext{
${ }^{1}$ According to Appdata.com, Zynga had 246 million monthly users, with Electronic Arts and Playdom commanding 49 million and 20 million monthly users respectively.
}

causing their desire to rise and the process of striving to begin again [8]. The baseline return is usually explained by people's happiness being relative to the others around them. For example, a graduate student spends all her time dreaming about her first tenure-track job, and she feels a peak of happiness when she finally secures it. However, our new professor now moves in social circles where all her friends have tenure, leaving her feeling like she's not doing as well as her friends, causing her happiness to return to the baseline.

\subsubsection{Loss aversion}

Loss aversion is the observation that people are more upset by loss than they are made happy by gain. As an example, someone who is given $\$ 100$ will gain less satisfaction than someone else who has $\$ 100$ taken away from them will lose [17].

\subsubsection{Reciprocal altruism}

Reciprocal altruism observes that, even if one were to ignore possible motivations for altruism itself (detailed in [34]), people will often respond to an action in kind: altruism with altruism, hostility with hostility and so on [5].

\subsubsection{Sunk cost fallacy}

Sunk costs are costs which have been incurred and can't be recovered, such as buying a membership to a tennis club. The member injures his arm, so shouldn't continue attending, but exclaims "I don't want to waste my membership fee!" and continues to play, hurting his arm further. This small example shows how people are influenced by sunk costs (likely due to loss aversion), and will act irrationally when faced with them [33].

\subsection{Behavioral Psychology}

\subsubsection{Overview}

Behavioral psychology is a branch of psychology investigated in the early part of the 20th century, which analyzed the observable behaviors of people and animals, and led to various theories such as operant conditioning, where an organism alters its voluntary behavior due to some stimulus.

\subsubsection{Goal-gradient Hypothesis}

Goal-gradient hypothesis is the finding that as animals approach a reward, they expend more effort. This behavior leads to some interesting consequences. For example, people in a café reward program purchase more coffees they closer they are to getting their free reward coffee [18]. In games, progress bars encourage the same behavior [14].

\subsubsection{Schedules of Reinforcement}

Schedules of reinforcement are theories pertaining to how organisms react when a reinforcer is given after different periods. A reinforcer is an event that occurs in response to some action, and they can either be positive, such as a giving a food pellet, or negative, such as a loud noise. A number of schedules are detailed in [9], and here we present the ones we will use.

Fixed interval schedule A fixed interval schedule is one where the reinforcement is given after a period of time from the original response, regardless of what happens in between that time. An example of this is in Animal 
Crossing, where a player can plant an apple, wait a set amount of time, and then an apple tree will appear. This type of schedule is not particularly effective at increasing responses.

Avoidance fixed interval schedule This is a modification of the fixed interval schedule, where something negative happens after a fixed period of time unless a response takes place. This schedule leads to a slow, steady response to prevent something negative from occurring. Hopson previously saw these schedules in Ultima Online, where housing would degrade if players did not visit it regularly [14].

Variable ratio schedule This schedule is otherwise known as the slot-machine schedule: a certain probability of a reward given is set up, but it's unknown at which response it will pay out. This schedule is the one that produces the greatest amount of response in organisms, as that reward might occur on the very next press of the lever. This is the same schedule used in loot-based games such as World of Warcraft or Diablo, where an enemy is given a certain chance of dropping a certain item when killed, leading players to kill certain enemies again and again in order to get the desired item.

\subsubsection{Shaping}

Shaping is a process that uses rewards to train animals to perform more complex behaviors, and is commonly seen in training animals (such as giving a dog a treat when it sits down, then only giving the dog a treat when it sits and then lies down, then only giving the dog a treat when it sits, then lies down, then rolls over...) [30]. Shaping is a core element of operant conditioning. For more on this, Yee looks at shaping in Everquest [38].

\section{RELATED WORK}

\subsection{Social Network Games}

SNGs have become an object of close scrutiny in recent years. A team at Michigan State University have paid special attention to performing quantitative and qualitative sociological investigations into the SNG player base [32, 37]. Their study of player motivations indicate that players use SNGs as a means of maintaining relationships, and that those relationships provide meaning and incentive to the game actions taken. For example, one participant noted that seeing other players with a higher rank or better game areas encouraged them to play longer, while another found that gifting created a sense of community.

The study of social network games has been notable in Scandinavia, with Deturding, Järvinen and Björk working towards identifying a definition for social games [10] and the design patterns found in SNGs $[6,16]$. One of the most interesting findings of this group is that SNGs are not necessarily social, often having minimal interactions with other players, and gameplay spaces being partitioned off, causing Björk to refer to such games as "Massively Single-Player Online Games."

The most prolific publisher on behavioral economics and video games is Hamari, who has begun to analyze games with social elements from a behavioral economics perspective. In [12], he uses loss aversion to explain how losing crops in a social game causes people to return to the game (which we also note in this paper), as well as finding usages of techniques such as the endowment progress effect. In a separate article, Hamari and Järvinen look at how game mechanics in SNGs can be used for virality, retention and monetization [13].

\section{PATTERNS}

\subsection{Overview}

We studied various 'Ville games in detail: Farm Ville, Pioneer Trail (renamed from FrontierVille), Empires 85 Allies, Indiana Jones Adventure World (which we'll refer to as Adventure World for short), CityVille and CastleVille. We classify these as 'Ville games due to their gameplay similarity to Farm Ville, where players construct game worlds either as a means of attaining more resources or as a creative expression, much like games such as Sim City, The Sims, Harvest Moon and Animal Crossing. A number of other SNGs could also fit inside this categorization, such as The Sims Social, however we limit our discussion to the Zynga games for consistency.

Inside these games we look for what we have termed "motivational game design patterns." Design patterns were first suggested by Alexander [2] as a means of identifying common problems and their solutions. Björk and Holopainen defines game design patterns as "commonly recurring parts of the design of a game that concern gameplay" [7]. This expands the original Alexander usage from just problemsolution pairs to include patterns that are less precise or that support creative experimentation. We alter Björk and Holopainen's definition slightly to create motivational game design patterns, which we define as commonly recurring parts of the design of a game that motivate some behavior in a player. For example, the neighbor bar (described in 4.5.3) motivates competitive players to improve their play space. However, we do not include patterns that facilitate reaching the motivated goal. Improving play spaces is facilitated by the ability to place buildings and decorations. We analyze how the neighbor bar pattern creates this motivation, but disregard the buildings and decorations from our study. We identified four main categories of patterns. Game space patterns relate to the virtual world in which the game takes place; progression patterns reflect how a player progresses in the game; reward patterns deal with how player actions are rewarded and social patterns describe how players interact with one another.

All the patterns can be seen in Table 1 .

\subsection{Play space}

\subsubsection{Harvesting}

Harvesting is an action in which a player expends some resource, that resource is locked away and changes in value, and is then redeemed later. The resource redeemed is not necessarily of the same resource as expended. The archetypal version of this action is planting crops: crops require seeds, which are sown into a patch of land, and grow into crops which are harvested for coins, supplies, or both. However, crops are not the only presentation used: Adventure Land sends out supply boats, and Empires 8 Allies uses depots such as lumber mills and ore mines to generate dif- 


\begin{tabular}{|l|l|l|l|}
\hline Category & Pattern & Theory & Other Examples \\
\hline Play space & Harvesting & Fixed interval schedule & Animal Crossing \\
\hline Play space & Withering & $\begin{array}{l}\text { Sunk cost fallacy, avoidance interval } \\
\text { schedule }\end{array}$ & Ultima Online \\
\hline Progression & Player level system & Goal-gradient hypothesis & Role-playing games \\
\hline Progression & Quests & Goal-gradient hypothesis, shaping & Role-playing games \\
\hline Rewards & Collections & $\begin{array}{l}\text { Intrinsic motivation, material cul- } \\
\text { ture }\end{array}$ & Bioshock, Skyrim \\
\hline Rewards & Currencies & Anchoring & Microsoft Points \\
\hline Rewards & $\begin{array}{l}\text { Extrinsic reward for } \\
\text { clicking }\end{array}$ & Variable ratio schedule & World of Warcraft \\
\hline Rewards & Returning bonus & Loss aversion & World of Warcraft \\
\hline Social & Altruistic actions & Reciprocal altruism & $\begin{array}{l}\text { Animal Crossing, } \\
\text { Forza Motorsport 3 }\end{array}$ \\
\hline Social & $\begin{array}{l}\text { Max-level AI neigh- } \\
\text { bor }\end{array}$ & Contrast effect & Metroid \\
\hline Social & Neighbor bar & Hedonic treadmill & Leaderboards \\
\hline
\end{tabular}

Table 1: Design patterns in 'Ville games, with their behavioral theories and previous sightings.

\begin{tabular}{|l|l|l|l|l|l|l|}
\hline Item & Time to grow & Costs & Produces & Production pm & Cost pm & Production : Cost \\
\hline Corn & 5 & 25 & 100 & 20 & 5 & 4 \\
\hline Watermelon & 240 & 240 & 625 & 2.60 & 1 & 2.60 \\
\hline Pineapple & 1440 & 576 & 1500 & 1.04 & 0.40 & 2.60 \\
\hline
\end{tabular}

Table 2: The cost and production of crops in Empires $\&$ Allies. Time is in minutes, pm stands for "per minute."

ferent types of resources. For ease, we'll refer to this action using the crops aesthetic.

When the player engages in a harvesting action, they are asked what seeds they would like to plant. Each seed costs a certain amount, takes a certain amount of time to reach fruition, and then produces a certain amount of goods. Table 2 looks at the numbers of planting crops in Empires 8 Allies. Note that to grow finances as quickly as possible, it is best to plant corn, as that generates 20 coins per minute. However, the player must always be playing at five minute intervals to maximize return. If the player wants to build their finances more slowly, but with less effort, they can invest in watermelons or pineapples. However, note that both watermelons and pineapples produce coins in the same ratio. If a player wanted to minimize her interaction, pineapples are a better crop than watermelons.

'Ville games always offer different pay-offs depending on how much time is required for the crop to grow. Here, corn supports players who wish to min-max their game, but they will likely not have enough in-game energy to perform such a task for long, and will need to pay money in order to progress with this tactic. We see that for more relaxed players, longer schedules provide the same pay off as shorter ones. While their actual time playing the game may be similar, or smaller, than those who interact with it more frequently, they remain engaged with the game for a longer calendar time.

Using real-time as a factor in crop growth shows that we are dealing with a fixed interval schedule, which primes players to return to the game. However, it is likely that this schedule is not the primary reason for returning to a crop, but players return due to the withering mechanic, discussed in the next section.

\subsubsection{Withering}

Of all the patterns in the "Ville games, withering is one of the most fascinating. Withering occurs when a crop is not harvested within a set period of time. Once that time passes, the crop spoils, and no longer pays out as much as it would have done had it been harvested in a timely manner.

In Table 3, three examples from different 'Ville games are presented. We can see how the punishment for withering has changed over the years. In Farm Ville, withering not only causes the player to lose the cost of the crop, but an extra punishment of 15 coins is levied when the withered crops are cleared. In Empires \& Allies, withered crops pay back half of what the seeds cost. However, in CastleVille, Zynga's latest game at the time of writing, the withered crops are still worth more than the cost of the seeds. Only after we put these numbers into our spreadsheet did we realize this was the case; the UI prompting of the withered crops alone (plus, perhaps, experience from more punishing games) led us to believe that money was lost from withered crops in Castle Ville.

The power of the withering pattern is due to the sunk-cost fallacy. Here, we can see that crops are a sunk-cost: once a crop is planted, the money for it can only be retrieved by returning at the right time, whether the player wants to or not. If the player would rather not play the game and see a movie instead, returning to the game is an irrational choice as it is a waste of their time. However, the sunk cost fallacy indicates that players are likely to feel a strong influence to return to the game and harvest their crops before they wither.

When looking at withering with the lens of behavioral psychology, we see another facet of the pattern. Note how we spoke about how harvesting sets up a fixed interval schedule for the player to respond to. The addition of withering 


\begin{tabular}{|l|l|l|l|l|l|}
\hline Game & Crop & Cost & Harvested Value & Withered Value & Gain from withered \\
\hline FarmVille & Black Tulips & 50 & 200 & -15 & -65 \\
\hline Empires \& Allies & Pineapples & 576 & 1500 & 288 & -288 \\
\hline CastleVille & Wheat & 100 & 550 & 275 & 175 \\
\hline
\end{tabular}

Table 3: The cost and losses/gains from withered crops.

means this schedule can be construed as an avoidance fixed interval schedule.

At this point, we see that we have two possible schedules for harvesting and withering, one where the player is attempting to get a reward, and one where the player is attempting to avoid a punishment. We hypothesize that different players may respond to each one in different proportions. For some, the possibility of gain may be a big motivator, whereas for others, the possibility of loss may be the primary driver.

\subsection{Progression}

\subsubsection{Player level system}

Each of the 'Ville games has a fairly standard player level system. As the player performs actions, experience points stars appear, which are collected to increase levels. Increased levels in the 'Ville games allow access to more powerful objects, usually in the form of increased production, which are valuable as usable space is often difficult to acquire.

Importantly, the player is given continual feedback about her current level with a UI element that shows a progress bar to the next level. As more experience points are gained, the bar increases towards the end, when the next level is unlocked. This feedback will most likely to lead to goalgradient hypothesis behavior, encouraging players to play more towards the end of the level. To increase player participation, the 'Ville games could utilize the endowment progress effect, giving more XP early on in a level to artificially inflate the progress bar.

\subsubsection{Quests}

Quests are tasks given by the game for a player to complete, with some reward offered for successful completion. Quests serve two purposes. Firstly, they provide another form of progress feedback, again pointing towards the effects of the goal-gradient hypothesis. Secondly, they provide a means of training the player to perform more complex game tasks. Quests start off easily completable (such as "Harvest four wheat bushels"), and players are shown various aspects of the game. As they learn the basics, the quests start asking for more difficult tasks (such as "Bake three loaves of bread," which requires wheat, grinding it into flour...). The length of time each new task takes steadily becomes longer and longer. Here we see a clear case of shaping, as the player is trained to perform more complex actions.

\subsection{Rewards}

\subsubsection{Collections}

Collections are items which are collected, but individually fulfill no gameplay task. Collection items will randomly appear when performing action (say, a bug that appears from clearing grass), and completing a collection usually results in a small bonus or a craftable item.
Neither behavioral economics nor behavioral psychology have much to say about collections, indicating that they may play a different role in the games than most of the patterns described in this paper. The fascinating property of collections is that they were valuable to players without conferring gameplay value, at a time when completing a collection didn't even result in a reward ${ }^{2}$. Reiss theorizes that collecting is a core intrinsic motivation [26], but developers have increasingly added extrinsic rewards for collecting. As we discuss in section 5, we hypothesize that collections are one of the active ways that players engage in the theme of the game. This sort of pattern is also in visible in games such as Animal Crossing and Skyrim and may indicate that material culture (how we culturally confer meaning to an object and relate to each other and ourselves is through material objects) not only exists in the real world, but in game worlds too [21].

\subsubsection{Resources}

In the 'Ville games, there are a number of resources. We define items as being a resources if they can be exchanged for a different item, for example, energy and a cow can be exchanged for milk. We have identified five different common currencies:

In-game money The coins created in-game.

Exclusive money This is the resource which is usually purchased with real money, but is sometimes rewarded in small increments during normal gameplay.

Energy Energy is a resource that governs how many actions a player can perform in a certain time period. Energy is purchasable using exclusive money.

Neighbors Many buildings, such as a Maiden's Tower in CastleVille, require staff. Staffing can happen in two ways: either players can pay exclusive money to fill spots with artificial agents, or a number of unique neighbors can staff the building, hence neighbors act as a resource. This is, perhaps, an explicit form of the theory of social capital, which states that social networks have a value much like other objects [25].

Game-specific resource Game-specific resources are game currencies, in addition to in-game money, that are used. Often these are building blocks used for creating items, like logs or ore.

While having a number of resources is not necessarily interesting in and of itself, there are a number of interesting aspects to how they are converted, particularly in-game money and exclusive money. Let's look at how CastleVille money exchanges. 15 crowns (the exclusive money) costs $\$ 2.00$,

\footnotetext{
${ }^{2}$ The value of collections was described by John Romero in a talk given to the IGDA Silicon Valley, noting they strongly appeal to a subset of players.
} 
leading to a conversion rate of $\$ 0.133$ to a crown. 1200 coins (the in-game money) also cost $\$ 2.00$, which equals $\$ 0.00166$ to a coin. These are not numbers that are easily remembered, and performing the conversions requires a great deal of cognitive effort.

All items in games have no market value until a designer assigns an initial cost. Let's look at "Jester's pants", which are worth 7000 coins. Now, one can also purchase a similar looking set of "Lederhosen", which are 2 crowns. What's the better deal?

It's actually very difficult to decide which is the best choice due to anchoring. It's hard to shake the feeling that the lederhosen is the better deal here, as 7000 is clearly a lot larger than 2. All the "Ville games do this: the in-game money is dealt in orders of magnitude greater sums than the exclusive money, giving the impression that the items which cost exclusive money are a better value, even though that's the resource that requires real money payment.

The final twist in the tale is that if we were to buy the required resources for each item at the exchange rate listed, the Jester's pants cost about $\$ 11.62$. The lederhosen cost $\$ 0.26$. Why is there such a large disparity, particularly when we just hypothesized that it's the lederhosen Zynga would like to sell? Well, partly the Jester's pants are technically purchasable from playing the game alone, so they are, in some sense, free (for many, particularly younger players, the "time is money" axiom does not hold). The other possibility is that the Jester's pants might not exist to be purchased. If we see the Jester's pants as a simple means of creating an anchor, they may well induce players that otherwise would not have purchased the exclusive money to buy some and purchase the lederhosen. Such a technique has been used successfully in restaurants for some time [31].

\subsubsection{Click reward}

We give the name "click reward" to the phenomenon that essentially all game actions in "Ville games result in some sort of reward appearing, usually in the form of XP stars and coins. There is much debate about the value of this extrinsic reward (rewards given for a task) versus that of intrinsic motivation (the internal gratification felt for simply performing the task), and this issue requires its own paper to properly address.

Click reward uses a variable ratio schedule for offering certain items: perhaps a chicken will provide chicken meat, but only in one out of ten clicks. If the meat is required for a quest completion, which combines this with goal-gradient hypothesis, an almost irresistible draw to clicking the chicken to attain the meat is created.

\subsubsection{Returning Bonus}

The returning bonus is a reward given for coming back to the game for consecutive days. Each day the bonus gets steadily greater, and the player is shown that there will be a bonus the next day, but its specific identity is not revealed. This plays to loss aversion, as the player feels like the bonus has already been endowed to them and will be lost if they don't return. The returning bonus is also a form of fixed interval schedule.

\subsection{Social}

\subsubsection{Altruistic actions}

Altruistic actions were defined by Björk as "Actions that have only explicit benefits for somebody else than is performing the action" [6]. This pattern appears throughout the "Ville games via gifting and visiting.

Gifting is the process of sending an item from one player to another. Often, the item is requested by the receiver as it is needed to finish a task, and the item can only be received as a gift, rather than found in the game world. Notably, the sender cannot find the item in the game world either. When the sender sends a gift (which she doesn't have), the game creates one and then sends it, thus the sender loses no item. When the gift is received, the 'Ville games will often prompt the receiver to send a gift back (also not taken from the receiver), setting up a reciprocal altruism gifting loop where neither player loses anything in the exchange. Worth noting is that, on both Facebook and Google+, the initial gifting request occurs in the open, creating an advertisement for the game each time. Once in a gifting loop, gifting between two players usually occurs within the game, removing the friction required to post on the social network itself.

Visiting is a mechanic where players can visit their neighbors' game spaces, and perform tasks. Some tasks, such as reviving withered crops, would often cost exclusive money for the host to perform herself. In addition, all the performable tasks would require the host to expend energy were she to perform the tasks herself, so the visit is always wellreceived. The visitor also receives rewards from visiting and performing actions, so often a visit is not entirely altruistic.

As all of the altruistic actions available are easily reciprocated, strong ties between players are created which require them to co-operate with one another, fostering a sense of belonging [37].

\subsubsection{High-level neighbor}

In all of the 'Ville games, with the exception of Farm Ville, players are given a computer-controlled neighbor for free, which the player is requested to visit during the tutorial stages of the game. Theoretically, this is to show players how to visit one another's spaces and perform altruistic actions, but it also serves another purpose. The neighbor's game space is often exceptionally designed, filled with premium items that cost hundreds of dollars to attain. When the player returns to her spartan game space, it feels even more empty and devoid of excitement than before, illustrating the power of the contrast effect. The contrast effect here might well inspire a player to play in order to build towards the high-level game space, and may even compel her to purchase exclusive money in order to attain a must-have item that she just saw.

\subsubsection{Neighbor bar}

The neighbor bar appears in all 'Ville games. Below the main viewing area is a list of all the player's game neighbors; friends from the social network that they have connected with in the game. The list is ranked by a player's level, with the list working from the lowest-level on the left, to the highest on the right. As a player gains levels, she moves up the list. This is essentially a leaderboard, albeit one that provides interactive elements, such as allowing players to visit their neighbors. The leaderboard drives competitive 
players onto a hedonic treadmill. Note the treadmill cannot be finished: the high-level neighbor is always at the top of the bar.

\section{DISCUSSION}

In this paper, we've not only identified a number of 'Ville design patterns, but a number of ideas from behavioral economics and behavioral psychology that relate to them. Are we now in a position to explain Cow Clicker?

There are very few gameplay mechanics in Cow Clicker, but those that do exist incorporate ideas we have looked at: the click countdown timer is a fixed interval schedule, players can collect different cows (encouraged by Bogost invoking scarcity, as he regularly cycled the cow designs), and they can act in a reciprocal altruistic relationship by placing each other's cows into their pastures (every click given to a player's cow results in a click on each cow in her pasture).

Reducing to just these few elements, Cow Clicker seems to take almost all the gameplay out of its game, even more than SNGs are accused of doing - leaving something that accentuates points for their own sake in a manner that recalls Margret Robertson's musings about gamification [27]. One possible conclusion to draw is that one or all of these patterns are so powerful that they can motivate a minority of people to return to a game that is at best tedious, and at worst abusive. And certainly Cow Clicker and other 'Ville games seem to indicate that there is inherent engagement to be found in these patterns, even if it is different from that traditionally discussed in game design writings. However, Cow Clicker may point to more.

Frank Lantz argues that "If this is a Skinner box, there's no cheese... There's just a button. I don't think mice push buttons that aren't hooked up to anything" [20], which suggests that Cow Clicker is not entirely explicable with behavioral lenses. However, he also notes that the "joke is obvious, broad, hilarious. Ian... was indefatigable at adding new jokes." Others have argued that most players at the end weren't in on the core parody [3], but the continuallyupdating humor was obvious whatever one's ironic distance from 'Ville games, never failing to offer a new and terrible cow-based pun. This suggests two things. First, that even as stripped-down an SNG as Cow Clicker is something more than its Skinner box. Second, perhaps what the Skinner box button is hooked up to is not within the game at all, but to the metagame that Bogost spent a great deal of time creating, just like all successful SNG developers.

In 4.4.1 we have already discussed one important way that all 'Ville games exceed the simple Skinner box model: collections. Another central way in which all 'Ville games do this is through decorating. Neither of these has a powerful impact on quantifiable game rewards, but both are central design elements and player activities. We hypothesize that these are important because they represent active ways of engaging a game's theme - which is also a pleasure found in activities that confer greater in-game rewards (e.g., quests). Far from avoiding this design approach for 'Ville games, Cow Clicker epitomized it, and only when Bogost removed the cows was this combination of (simple) motivational patterns and theme engagement disturbed.

Beyond this, more so than games on other platforms, the value of SNGs to players isn't contained within the games themselves, but as part and parcel of the relationship building and maintenance that Wohn et al. noted [37]. What fun is Parking Wars without the opportunity to boast at the watercooler? Our framework provides us with a means of analyzing the game mechanics, and how those mechanics support social interactions, but in the case of Cow Clicker many of those interactions occurred externally from the game itself. In contrast, most SNGs attempt to force certain social contacts to happen in-game by presenting the user with dialog boxes encouraging game invites or sharing rewards. In either case, social interactions around SNGs are clearly a central aspect of the pleasure of the experience for many players.

Which leads us to the conclusion that Cow Clicker so successfully epitomized the 'Ville games it set out to parody that we can use it to guide an analysis of how these games operate. The core game systems are based on the motivational game design patterns identified in this paper. But these do not fully capture the metagame social relationship aspects, which are key, or the network effects that come from the large numbers already playing Zynga games. Within Zynga games, it may be that what differentiates the 'Ville games from one another is simply their theme, and certain themes engage broader sets of the population than others, with the theme being continually reinforced by the collecting and decorating mechanics. Our framework doesn't explicitly take into account the overlaid theme, much like other game analysis tools like Machinations [11] or MDA [15], but it is clear that it cannot be ignored entirely.

\section{CONCLUSION}

While SNGs are relatively simple in comparison to other digital games, they encompass many of the motivational techniques identified by behavioral economics and behavioral psychology. Knowingly or not, they deftly incorporate these techniques to motivate players, and utilize a wide array of phenomena to appeal to as broad an audience as possible. Our framework allows us to identify motivational game design patterns, which encourage players to return to a game again and again.

Scholars have collated a wealth of game design patterns which designers can draw upon, but little time has been spent ascertaining what makes one design better than another. Future work could continue linking game design patterns to observations of humans in other fields, so that we can better understand how the effective patterns work, and how less effective patterns can be improved. Investigating this relationship may also lead us to derive new game design patterns based on unexplored psychological theories.

We believe bringing fields such as behavioral economics and behavioral psychology together with game design will lead us not only to a better understanding of games, but a better use of game design patterns, and ultimately, happier, more engaged, players.

\section{ACKNOWLEDGMENTS}

The authors would like to thank Dan Friedman and Steve Whittaker from UC Santa Cruz for reviewing our usage of behavioral economic and behavioral psychology theories.

\section{REFERENCES}

[1] 2010 Social Gaming Research. Tech. rep., Information Solutions Group, 2010. 
[2] Alexander, C. A Pattern Language, vol. 21. Oxford University Press, 1977.

[3] Alexander, L. The Life-changing $\$ 20$ Rightward-facing Cow. Kotaku (2011). http://goo.gl/D2Equ.

[4] Ariely, D. Predictably Irrational, Revised and Expanded Edition: The Hidden Forces That Shape Our Decisions. Harper Perennial; 1 Exp Rev edition, 2010.

[5] Axelrod, R., and Hamilton, W. D. The Evolution of Cooperation. Science 211, 4489 (1981), 1390-1396.

[6] BJÖRK, S. Principles and patterns of social games: Where's the difference compared to other games? In GCO Games Convention Online 2010 (2010). http://bit.1y/chZdiJ.

[7] Björk, S., And Holopainen, J. Patterns in Game Design (Game Development Series), 1 ed. Charles River Media, Dec. 2004.

[8] Brickman, P., And Campbell, D. T. Hedonic relativism and planning the good society. In Adaptation level theory A symposium, M. H. Appley, Ed. Academic Press, 1971, pp. 287-302.

[9] Chance, P. Learning and Behavior. Wadsworth Pub Co; 4th edition, 1998.

[10] Deterding, S., Björk, S., Dreyer, S., Järvinen, A., Kirman, B., KüCKlich, J., PaAvilainen, J., RaO, V., And Schmidt, J.-H. Social Game Studies: A Workshop Report. Tech. Rep. 2010, 2010. http://socialgamestudies.org/report.

[11] Dormans, J. Engineering Emergence: Applied Theory for Game Design. PhD thesis, University of Amsterdam, 2012.

[12] Hamari, J. Perspectives from behavioral economics to analyzing game design patterns: loss aversion in social games. In Proceedings of the CHI 2011 Social Games Workshop (2011).

[13] Hamari, J., AND Järvinen, A. Building Customer Relationship through Game Mechanics in Social Games. In Business, Technological, and Social Dimensions of Computer Games: Multidisciplinary Developments. 2011, p. 18.

[14] Hopson, J. Behavioral Game Design. Gamasutra (2011).

[15] Hunicke, R., LeBlanc, M., and Zubek, R. MDA: A formal approach to game design and game research. In Proceedings of the Challenges in Game AI Workshop, 19th National Conference on Artificial Intelligence (San Jose, CA, July 2004), pp. 1-5.

[16] Järvinen, A. Game design for social networks: interaction design for playful dispositions. In Proceedings of the 2009 ACM SIGGRAPH Symposium on Video Games - Sandbox '09 (New York, New York, USA, Aug. 2009), ACM Press, p. 95.

[17] Kahneman, D., And Tversky, A. Prospect Theory: An Analysis of Decision under Risk. Econometrica: Journal of the Econometric Society (Nov. 1979).

[18] Kivetz, R., Urminsky, O., And Zheng, Y. The Goal-Gradient Hypothesis Resurrected: Purchase Acceleration, Illusionary Goal Progress, and Customer Retention. Journal of Marketing Research 43, 1 (Feb. 2006), 39-58.
[19] Koster, R. A Theory of Fun for Game Design, 1 ed. Paraglyph Press, Nov. 2004.

[20] Lantz, F. [Insert Cow Pun Here]. http: //gamedesignadvance. $\mathrm{com} / \mathrm{?}$ = $=2383$.

[21] Lehdonvirta, V. Online spaces have material culture: goodbye to digital post-materialism and hello to virtual consumption. Media, Culture \& Society 32, 5 (Sept. 2010), 883-889.

[22] Lowenstein, G., And Ubel, P. Economics Behaving Badly. New York Times (2010).

[23] McGonigal, J. Reality Is Broken: Why Games Make Us Better and How They Can Change the World. Penguin Press HC, The, 2011.

[24] Nunes, J. C., AND Drèze, X. The Endowed Progress Effect: How Artificial Advancement Increases Effort. Journal of Consumer Research 32, 4 (2006), 504-512.

[25] Putnam, R. D. Bowling Alone: The Collapse and Revival of American Community. Touchstone Books by Simon \& Schuster; 1st edition, 2001.

[26] Reiss, S. Multifaceted Nature of Intrinsic Motivation: The Theory of 16 Basic Desires. Review of General Psychology 8, 3 (2004), 179-193.

[27] Robertson, M. Can't play, won't play, 2010. http://goo.gl/Cc1tY.

[28] Rollings, A., And Morris, D. Game Architecture and Design: Learn the Best Practices for Game Design and Programming. Coriolis Group Books, 1999.

[29] SChell, J. DICE 2010: "Design Outside the Box". Tech. rep., DICE, Las Vegas, 2010. http://goo.gl/yWKyp.

[30] Skinner, B. F. Science And Human Behavior. Free Press, 1965.

[31] Speiser, M. What We Can Learn About Pricing From Menu Engineers, Sept. 2009. http://goo.gl/4HrJE.

[32] Sung, J., Buornrud, T., LeE, Y.-H., And Wohn, D. Y. Social network games: Exploring Audience Traits. In Proceedings of the 28th of the international conference extended abstracts on Human factors in computing systems - CHI EA '10 (New York, New York, USA, Apr. 2010), ACM Press, p. 3649.

[33] Thaler, R. Toward a positive theory of consumer choice. Journal of Economic Behavior \&s Organization 1, 1 (Mar. 1980), 39-60.

[34] Thaler, R. The Winner's Curse: Paradoxes and Anomalies of Economic Life. Princeton University Press, 1994.

[35] Tversky, A., And Griffin, D. Endowment and contrast in judgments of well-being. Subjective well-being: An interdisciplinary perspective.

[36] Vogt, P. Cow Clicker Founder: If You Can't Ruin It, Destroy It. NPR (2011). http://goo.gl/uJznS.

[37] Wohn, D. Y., Lampe, C., Wash, R., Ellison, N., AND VitaK, J. The "S" in Social Network Games: Initiating, Maintaining, and Enhancing Relationships. In 2011 44th Hawaii International Conference on System Sciences (Jan. 2011), IEEE, pp. 1-10.

[38] YEE, N. The Virtual Skinner Box. http://www.nickyee.com/eqt/skinner.html. 